\title{
Dust Temperatures and Opacities in the Central Parsec of the Galactic Center Modeled from Analysis of Multi-wavelength Mid-infrared Images
}

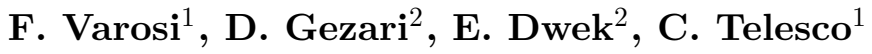 \\ ${ }^{1}$ Univ. of Florida-Gainesville, USA email: varosi@astro.ufl.edu \\ ${ }^{2}$ NASA/GSFC, Greenbelt, MD USA
}

We have analyzed multi-wavelength mid-infrared images of the central parsec of the Galactic Center using a two-temperature line-of-sight (LOS) radiative transfer model at each pixel of the images, giving maps of temperatures, luminosities and opacities of the hot, warm, cold (dark) dust components. The data consists of images at nine wavelengths in the mid-infrared (Nband and Q-band) from the Thermal Region Camera and Spectrograph (T-ReCS) instrument operating at the Gemini South Observatory. The results of the LOS modeling indicate that the extinction optical depth is quite large and varies substantially over the FOV.

The high-resolution images of the central parsec of the Galactic center region were obtained with T-ReCS at Gemini South in January 2004. These images provide nearly diffraction-limited resolution $\left(\sim 0.5^{\prime \prime}\right)$ of the central parsec. The T-ReCS images were taken with nine filters $(3.8$, $4.7,7.7,8.7,9.7,10.3,12.3,18.3$ and $24.5 \mu \mathrm{m}$ ), over a field-of-view (FOV) of $20 \times 20$ arcsec.
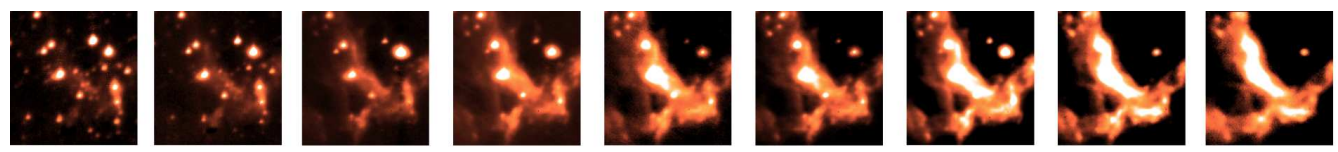

Figure 1. Nine images of the central parsec obtained with T-ReCS (Thermal Region Camera and Spectrograph) at Gemini South in January 2004. These images are aligned and assembled into a stack to extract the mid-IR SED.

We applied the new two-temperature version (Varosi 2015) of our planar, line-of-sight (LOS), radiative transfer dust model (Dwek and Varosi 1998) to each mid-infrared nine-image stack. Attempts to fit both mid-infrared and near infrared data with a single dust temperature did generally do not yield satisfactory fits. The two-temperature LOS model is a better approximation of the true source morphology. In the model, the dust is actually arranged as three components along the LOS, i.e., 1) the hot emitting dust at the source, 2) the warm emitting dust near the source, and 3) a third component where a fraction of the cold emitting dust is a foreground absorbing layer along the LOS.

The LOS model maps of the distribution of physical conditions show the following results: The hot dust $\left(200 \mathrm{~K}<T_{\text {hot }}<600 \mathrm{~K}\right)$ component inferred by LOS model is from both diffuse emission and emission close to embedded sources. The very hottest dust $(>400 \mathrm{~K})$ is found along ionization front around central cavity (Sgr $\mathrm{A}^{*}$ ) and around IRS-3. This dust appears to be mostly heated by HeI emission line stars and by the embedded source in IRS-3. The mass of hot dust in the observed field is $>4 \times 10^{-3} M_{\odot}$ and the peak density occurs near IRS- 1 . The warm dust $\left(60 \mathrm{~K}<T_{\text {warm }}<100 \mathrm{~K}\right)$ temperature peaks around IRS-10 and east of ionization front, while the density peaks around IRS-1. The mass of warm dust in the FOV is $>1 M_{\odot}$. The mass of cold (dark) dust in FOV is $>3 M_{\odot}$. The LOS extinction due to cold dust is highly non-uniform, varying by factor of 3 in FOV. The model infers a cloud of cold dust and gas in front of IRS-3 along the LOS with surface mass density $>1000 M_{\odot} / \mathrm{pc}^{2}$.

The discrete sources have typical dust temperatures of $280 \mathrm{~K}$. Those sources are best fit by a hot silicate emission, suggesting O-rich ejecta. There is a diffuse emission component with temperatures from $100-400 \mathrm{~K}$, with a clearly defined temperature gradient. The hottest dust lies along the ionization front around the central cavity (around Sgr A*), and may be heated both 

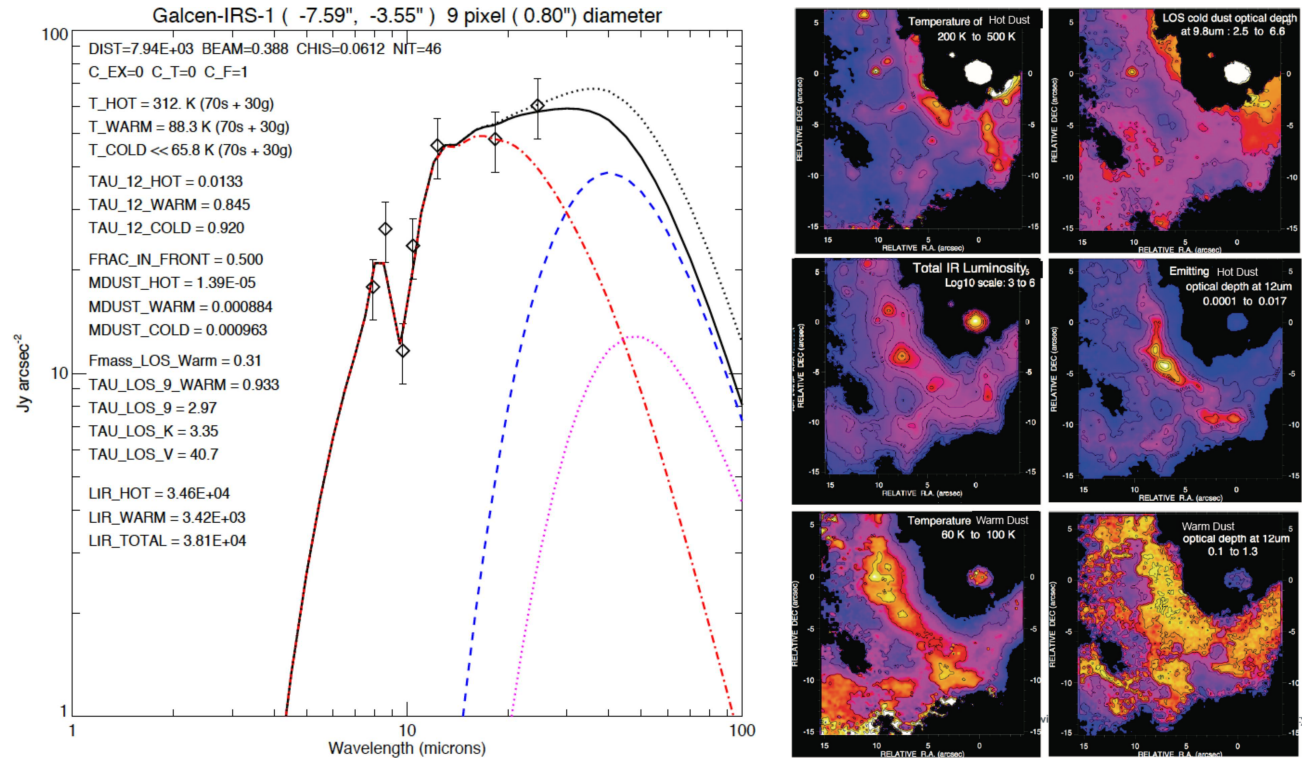

Figure 2. (Left) Model fit to the IRS-1 SED. The solid line represents the non-linear least-squares fit to the data points (diamonds). Also listed in the figure are the source luminosity, observed luminosity, dust temperature, intrinsic optical depth, and line-of-sight optical depth. (Right) The two-temperature model results for the SEDs extracted from the 9-image stack, for all pixel in the FOV: distributions of hot, warm and cold dust temperature, luminosity, observed luminosity, intrinsic source optical depth, and line-of-sight extinction optical depth.

by a distribution of HeI emission-line stars and unresolved embedded sources. The extinction to the GC is non-uniform, with $\tau_{9.8 \mu m}$ varying from 3 (near IRS-1) to 6 (near IRS-3), possibly due to molecular clouds of surface mass $>1000 M_{\odot} / \mathrm{pc}^{2}$. The total IR luminosity from the central parsec of the Galaxy is at least $4 \times 10^{6} L \odot / \mathrm{pc}^{2}$ in $\mathrm{FOV}=0.8 \mathrm{pc} \times 0.8 \mathrm{pc}$.

We conclude that most of the cold dust extinction is local to the central parsec. The large column densities of dust and gas, together with the clumpiness of the mid-infrared emission, suggest that the region has formed and will form many stars. The hot and warm emitting dust components produce high luminosities, and the overall results suggest that the heating is produced by embedded stars and distributed HeI stars.

\section{References}

Dwek, E. and Varosi, F. 1998, NASA/Goddard - Technical Report.

Varosi, F., 2015, private communication. 